\title{
Nanoparticle trapping and characterization using open microcavities
}

\author{
A. A. P. Trichet, ${ }^{* \dagger}$ P. R. Dolan,$^{\dagger}$ D. James, ${ }^{\ddagger}$ G. M. Hughes ${ }^{\dagger}$ C. Vallance,,$^{\ddagger}$ and \\ J. M. Smith ${ }^{\dagger}$ \\ Department of Materials, University of Oxford, OX1 3PH, UK, and Department of \\ Chemistry, University of Oxford, OX1 3RQ, UK \\ E-mail: aurelien.trichet@materials.ox.ac.uk
}

\begin{abstract}
Characterization and trapping of nanoparticles in solution is of great importance for lab-on-a-chip applications in biomedical, environmental and materials sciences. Devices are now starting to emerge allowing such manipulations and investigations in realtime. Better insights into the interaction between the nanoparticle and the optical trap is therefore necessary in order to move forward in this field. In this work, we present a new kind of nano-tweezers based on open microcavities. We show that by monitoring the cavity mode wavelength shift as the particle diffuses through the cavity, it is possible to establish both the nanoparticle polarizability and its coefficient of friction. Additionally, our experiment provides a deep insight in the interaction between the nanoparticle and the cavity mode. The technique has built-in calibration of the trap strength and spring constant, making it attractive for practical applications.
\end{abstract}

*aurelien.trichet@materials.ox.ac.uk

${ }^{\dagger}$ Department of Materials, University of Oxford, OX1 3PH, UK

${ }^{\ddagger}$ Department of Chemistry, University of Oxford, OX1 3RQ, UK 
This work illustrates the potential of such optical microcavities for future developments in nanoparticle sensors and lab-on-a-chip devices.

Keywords: Nanophotonics, optical trapping, nanotweezer, nanoparticle, microcavity, sensor

Within the last decade, optical microresonators have emerged as promising components for detection and characterisation of single nanoparticles. ${ }^{1-7}$ The combination of small mode volume and high finesse provides both strong interaction between the confined optical field and nanoscale particles and a high resolution 'ruler' with which to measure small mode perturbations. Electromagnetic field enhancement in resonators has also been used to optically trap nanoparticles. ${ }^{8-12}$ In particular, the strong intensity gradients that occur in the evanescent fields caused by surface plasmons, has enabled the trapping of small nanoparticles ${ }^{13,14}$ and even single proteins. ${ }^{15,16}$ Photonic crystals have been used to trap and monitor single virus interacting with antibodies ${ }^{17}$ demonstrating the maturity of these technologies.

Sensing and trapping of nanoparticles both rely on the same physical phenomenon, namely the dipolar interaction between the nanoparticle and the resonant optical modes within the cavity. The interaction leads to a shift in the resonance frequencies of the modes, providing a mechanism for particle sensing. It also results in an optical force on the nanoparticle in the direction of the intensity maximum of the cavity mode, providing a trapping mechanism. Both phenomena have already been combined within single nanostructures, resulting in a positive self-induced feedback mechanism ${ }^{18-20}$ between the intra-cavity field and the nanoparticle position. In terms of sensing, the nanoparticle-induced changes in the laser intensity transmitted through the nanostructures have been measured and correlated to the particle polarizability and coefficient of friction. ${ }^{21}$

Such trapping devices with sensing capabilities are on the verge of finding powerful applications in physical, chemical and biological sciences. However, a single characterization system that brings together in-situ detection, trapping and multiple quantitative measurements of the particle properties supported by a comprehensive theory is still to be demon- 
a) $\mu \mathrm{m}$

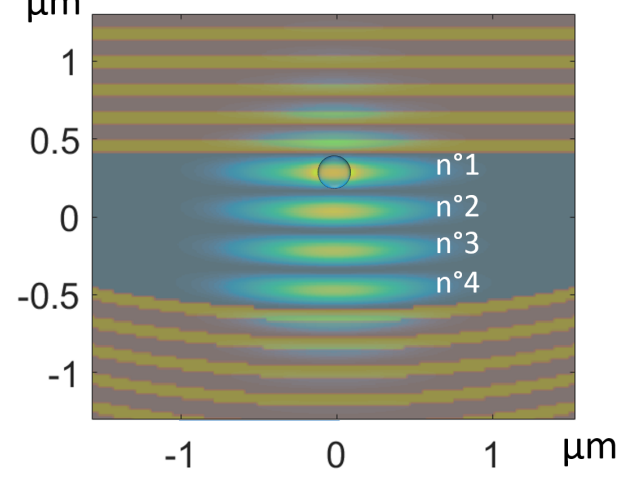

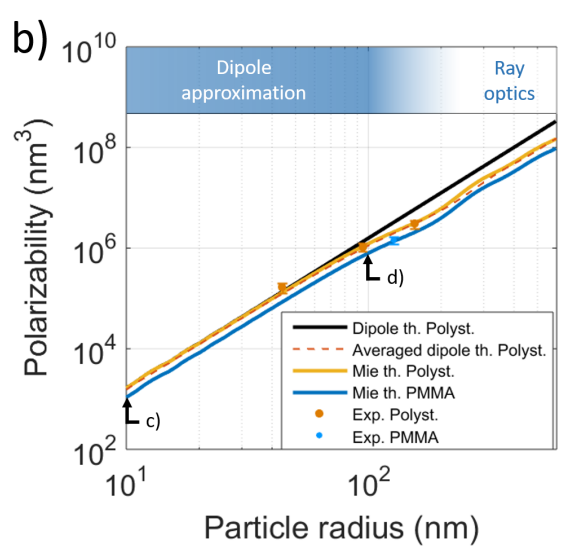

Figure 1: a) Open-access mirror structure and mode wavefunction at $640 \mathrm{~nm}$ obtained from FDTD simulations including the four nodes numbers. b) Theoretical polarizability within the dipole (black line), Mie (orange for polystyrene and blue line for PMMA) and averaged dipole (red dashed line for polystyrene) theories on a loglog plot. Experimental point in orange for polystyrene and in light blue for PMMA as a function of there nominal radius. c) and d) Normalized effective polarizability maps for a $10 \mathrm{~nm}$ and $100 \mathrm{~nm}$ radius nanoparticle respectively.

strated. Such a step is essential for the development of the field of nanotweezers in order to avoid experimental misinterpretations and artefacts and to cope with unknown or changing samples.

In the following, we show that open-access microcavities can be used as optical tweezers with in-situ force calibration and nanoparticle sensing capabilities, with no need for a priori knowledge of the particle properties. The shift in cavity mode frequency during a trapping event provides information on both the nanoparticle and trap properties, as well as on the trapping force holding the particle in the trap. We demonstrate the ability to determine both the nanoparticle polarizability, i.e. its optical response to an electromagnetic field, and its coefficient of friction. This work proved that open-access microcavities provide a robust platform for nanoparticle trapping and characterization.

\section{Open-access microcavities and nanoparticle polarizability}

Open-access microcavities exhibit similar mode structure to that of standard optical traps, and therefore provide a well-understood tool for nanoparticle trapping and sensing. The open 
microcavities used in the present work are made up of two opposed micron-scale mirrors (see Figure 1 a) ) with one mirror concave in order to provide a fully-confined optical mode. The mirror separation is controlled using a piezoelectric positioner, thus tuning the mode wavelength. Nanoparticles can directly diffuse to the region with highest electromagnetic field. In the present work, the concave mirror has a radius of curvature (RoC) of $4 \mu \mathrm{m}$, and the mirror separation is $\approx 1 \mu \mathrm{m}$, which leaves a $400 \mathrm{~nm}$ channel between the flat parts of the mirrors through which nanoparticles can diffuse into the trap. Our fabrication technique, described in the Methods section, offers excellent reproducibility, as well as nanometric control over the photonic potential. ${ }^{22,23}$ For the cavity length employed, the confined mode has four antinodes between the mirror surfaces, numbered from 1 to 4 on the Finite Difference Time Domain (FDTD) simulations shown in Figure $1 \mathrm{a}$ ). The mode volume $V$ is computed using this simulation to be $1.7 \times \lambda^{3}$ for a waist of $560 \mathrm{~nm}$, and the Quality factor Q, of the cavity mode is measured to be 18000 .

The mode shift $\Delta \lambda$ induced by the particle presence and used as the sensing mechanism can be written:

$$
\Delta \lambda(\vec{r})=-\frac{\lambda \alpha}{2 V} \times \widetilde{I}(\vec{r})
$$

where $\alpha$ is the polarizability of the nanoparticle and $\widetilde{I}(\vec{r})$ is the normalized mode intensity, ie. $\widetilde{I}(\vec{r})=1$ for a particle at the maximum intensity. Within the dipolar approximation, a nanoparticle with a radius $r$ and a refractive index $n_{N P}$ has a polarizability given by: ${ }^{1}$

$$
\alpha=4 \pi r^{3} \frac{n_{N P}^{2}-n_{w}^{2}}{n_{N P}^{2}+2 n_{w}^{2}}
$$

where $\mathrm{n}_{w}=1.33$ is the refractive index of water. This radius dependence of the polarizability for polystyrene particles $\left(\mathrm{n}_{N P}=1.58\right)$ is displayed in Figure 1 b) (black line). In this work, we have also used PMMA nanoparticles $\left(\mathrm{n}_{N P}=1.49\right)$. The dipole theory is a good approximation for particles with radius less than about $70 \mathrm{~nm}$, above which Mie theory 
results in a somewhat lowered polarizability (orange line for polystyrene and blue line for PMMA). A more simplistic understanding than offered by the Mie theory simulations is given by an averaged dipole theory (dashed red line) which involves integrating the dipole response over the particle volume (see Supplementary Information). The agreement with the Mie theory leads to the conclusion that the Mie resonances do not play a direct role in terms of the nanoparticle polarizability in our configuration. In addition to reducing the effective polarizability compared to a pure dipole model, this averaging effect also changes the trapping potential landscape seen by the particles, as shown in Figure $1 \mathrm{c}$ ) and d) for radius $10 \mathrm{~nm}$ and $100 \mathrm{~nm}$ respectively. The averaging effect softens the potential for particles above $r=50 \mathrm{~nm}$ and creates 'bridges' between the anti-nodes, allowing the particle to diffuse in between them for potential depths that are small compared to the thermal energy.

\section{Monitoring trapping events}

Using our optical setup as described in the Methods section and the Supporting information, we can optically trap a nanoparticle as well as monitor the cavity mode resonance. The spectra are acquired by scanning the flat mirror thanks to a piezoelectric actuator while shining a mode-matched fixed wavelength laser. These spectra are then calibrated and converted into a wavelength shift axis. Typical spectra are displayed in Figure 2 a). The blue and red circles in Figure 2 a) correspond to the transmission of the microcavities at $\mathrm{t}=0$ $\mathrm{s}$ and $\mathrm{t}=1.45 \mathrm{~s}$, respectively before and during a trapping event. The low and high intensity peaks correspond respectively to the reference and trapping cavity. The perturbation $\Delta \lambda(t)$ to the cavity mode solely due to the presence of a particle is then measured by subtracting the constant value of the mode shift before the trapping event $\Delta \lambda_{0}$ (ie. before $\mathrm{t}=0.3 \mathrm{~s}$ ) to the peak-to-peak distance fitted on all the spectra $\Delta \lambda_{0}+\Delta \lambda(t)$. Using this self-referencing technique in between a reference and a trapping cavity, we can greatly reduce the effect of mechanical noise on the measured mode perturbations and retrieve only the perturbation created by the nanoparticle $\Delta \lambda(t)$. On Figure 2 a), the measured peaks fit well to Lorentzian 
functions (dashed lines), reflecting the homogeneous cavity lineshapes and confirming that no mechanical instability or heating effects occur during each sweep through resonance. The time evolution of the cavity mode spectrum is displayed in Figure $2 \mathrm{~b}$ ). At time $t=0.3$ s a particle becomes trapped in the sensor cavity and its mode displays shifts of order 0.3 $\mathrm{nm}$ over a period exceeding 1.5 seconds. For comparison the free diffusion time $\tau_{\text {diff }}=\frac{V^{2 / 3}}{6 D}$ through a cavity mode of volume $V$ for a $200 \mathrm{~nm}$ diameter particle is about $80 \mathrm{~ms}$, as illustrated by the white line at the bottom left of the figure. The significant fluctuations in the mode shift of the sensor cavity during this time show that the particle continues to undergo Brownian motion within the trapping potential.

\section{Mode shift and scattering}

Once the mode shift has been extracted from Figure $2 \mathrm{~b}$ ), we can plot its evolution with time as shown in Figure 3 a). The shift baseline is determined before the particle enters the trap, and three standard deviations of this baseline suggests a minimum detectable shift of 0.9 pm. The polarizability of the particle is determined from the maximum mode shift observed. In the dataset displayed, a maximum mode shift of $370 \mathrm{pm}$ is measured. Averaging over 18 events, we found a mean maximum mode shift of $390 \mathrm{pm}$. Using the polarizability computed with the Mie theory for a $200 \mathrm{~nm}$ diameter nanoparticle, ie. $9.6 \times 10^{5} \mathrm{~nm}^{3}$, we can measure a mode volume of $3 \times \lambda^{3}$. The discrepancy with the mode volume obtained with FDTD is likely to originate from fabrication imperfections and deviations in the refractive index of the DBR's coating. Using this experimental calibration of the mode volume, we have measured the polarizability of few different types of nanoparticles. Mean polarizabilities of polystyrene $(\mathrm{r}=88 \mathrm{~nm}$ and $\mathrm{r}=300 \mathrm{~nm}$ in orange $)$ and PMMA $(\mathrm{r}=250 \mathrm{~nm}$ in light blue $)$ nanoparticles are displayed on Figure 1 b) and show excellent agreement with Mie theory. The errorbars gives the standard deviation over the measured events and originates from the radius polydispersion of the nanoparticle solution, ie. $\pm 10-15 \%$. These measurements demonstrate our system capability to measure the polarizability of a single nanoparticle. 

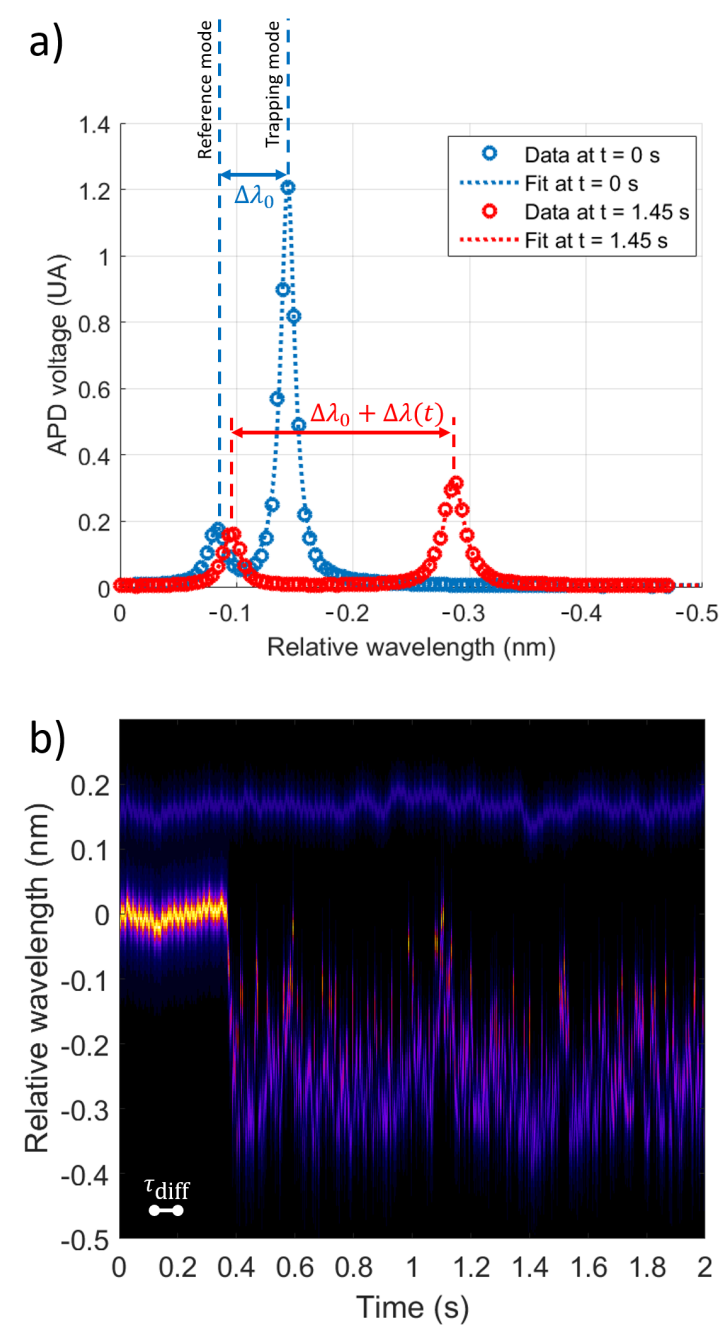

Figure 2: a) Transmission scan before $(\mathrm{t}=0 \mathrm{~s})$ and during $(\mathrm{t}=1.45 \mathrm{~s})$ a trapping event. Blue (red) circles are experimental data and blue (red) dashed lines are double Lorentzian fits. At $\mathrm{t}=0 \mathrm{~s}$, the trapping (reference) cavity is the high (low) intensity peak. b) Transmission scans as a function of time. The top (bottom) transmission peak is the reference (trapping) cavity. $\tau_{\text {diff }}$ gives the timescale of free diffusion of a $200 \mathrm{~nm}$ nanoparticle in the cavity volume.

The data in Figure 3 a) reveals that the maximum mode shift exhibits steps as a function of time (red line). We find that there are four different levels, originating from the movement of the particle between the four different field antinodes shown in Figure 1 a).

An interesting property of the cavities is revealed by recording the change in mode linewidth, which provides a measure of the rate at which the trapped particle scatters photons from the cavity mode. Figure $3 \mathrm{~b}$ ) displays a scatter graph of the line-width as a function of the cavity mode shift, with a colour scale relating to the density of points. We find that 
a)
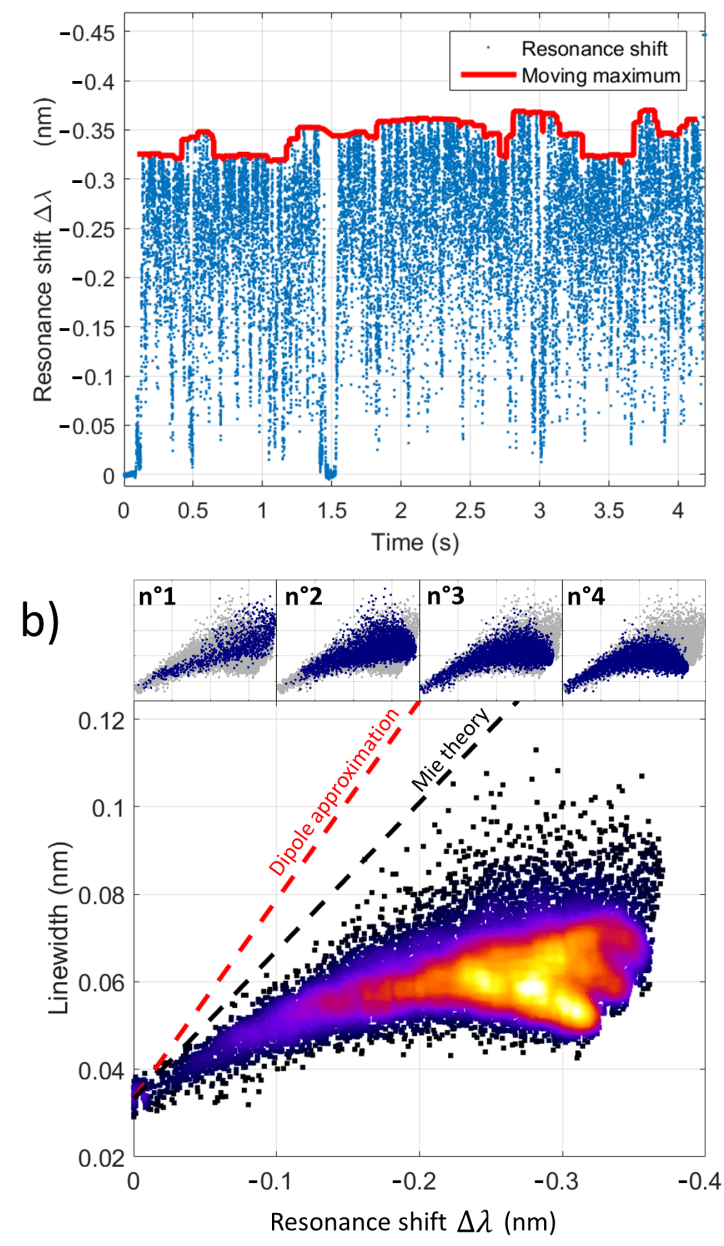

Figure 3: a) Blue dots: Experimental mode shifts versus time during a trapping event. The red line is the moving maximum over $80 \mathrm{~ms}$. b) Mode linewidth as a function of its shift for the same event. The colour scale indicates the density of points. The dipole (dashed red line) and Mie (dashed black line) theories. Insets: Same graphics but split over the four different branches.

the scattering that occurs from the particle in the cavity is significantly reduced by a factor $\beta_{f s}$ compared to a similar particle in free space using either dipole or Mie scattering theory (dashed lines). Consequently, the linear relation used to link mode shift and linewidth is rewritten: ${ }^{1}$

$$
\frac{\delta \lambda^{s c}}{\triangle \lambda}=-\frac{8 n_{w}^{3} \pi^{2} \alpha}{3 \lambda^{3}} \times \beta_{f s}
$$

This mismatch, displayed on Figure $3 \mathrm{~b}$ ), can be attributed to the fact that in a micro- 
cavity, the optical density of states into which a photon can scatter is reduced compared with a free-space environment. Based on the observed discrepancy, we estimate that the density of states accessible is reduced by as much as $\beta_{f s} \approx 65 \%$. Using FDTD simulations (see Supporting Information), we have computed a reduction of the available density of states of $\beta_{f s} \approx 60 \%$ in excellent agreement. It is also possible to observe four distinct 'branches' within the overall signal related to the four different antinodes of the mode. They can be separated using the moving maxima from Figure 3 a) (see insets of Figure 3 b) ). Each shows a distinct grouping of points on the scatter graph characteristic of the longitudinal position of the antinode in the microcavity. The antinode $\mathrm{n}^{\circ} 4$, closest to the concave mirror, displays the greatest suppression of scattering.

\section{Trap strength measurement}

The probability of occupation (PO) of each mode shift $\mathcal{N}$, extracted from the data sets in Figure 3 a), provides a measure of the trap strength. In terms of the normalized mode shift $\Delta \widetilde{\lambda}=\frac{\Delta \lambda}{\Delta \lambda_{\max }}$, the PO is given by :

$$
\mathcal{N}=\mathcal{N}_{0} \times \rho(1-\Delta \widetilde{\lambda}) \times e^{-f(1-\Delta \widetilde{\lambda})}
$$

where $\rho(1-\Delta \widetilde{\lambda})$ is the density of states (DoS), reflecting the number of particle positions that lead to the same shift; $e^{-f(1-\Delta \widetilde{\lambda})}$ is the Boltzmann factor, with $f$ a dimensionless measurement of the trap strength; and $\mathcal{N}_{0}$ is a normalization constant. The trap strength is the ratio of the trap depth to the thermal energy:

$$
f=\frac{U_{\max }}{k_{\mathrm{B}} T}=\frac{\alpha}{n_{w} c \epsilon_{0}} \frac{I_{0}}{k_{\mathrm{B}} T}
$$

The DoS is displayed in Figure 4 a) with the black solid and dashed lines showing the FDTD and Mie simulations, respectively. For comparison, the DoS for a parabolic trap is represented by the red solid line. The inset of Figure 4 a) emphasizes the non-parabolicity 

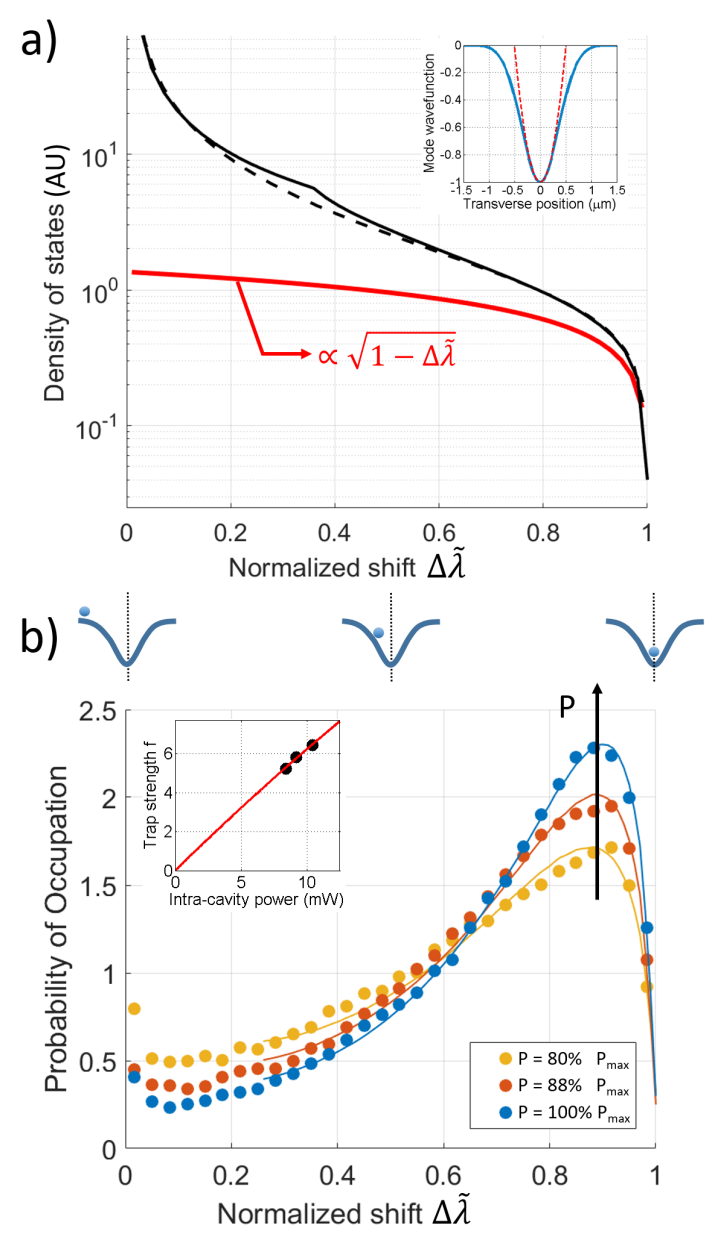

Figure 4: a) Theoretical density of states (DoS) on a logarithmic scale as a function of the normalized shift. Red lines: DoS for a parabolic trap. Black solid and dashed lines, respectively, the density of states obtained from Mie theory and FDTD simulation. b) Probability of Occupation (PO) as a function of the normalized shift for three different powers. Solid lines corresponds to the fit using equation 4 Inset: Trap strength $f$ as a function of power. The errorbars are given by the size of the dots. Solid line: best fit of equation 6 .

of the microcavity mode wavefunction. The small increase in the DoS predicted by Mie theory relative to the FDTD calculations for $\Delta \widetilde{\lambda} \approx 0.35$ is a result of the bridging between anti-nodes in the optical trapping potential.

The temperature $T$ appearing in equation 5 is expected to change linearly with the intra-cavity power $P$ :

$$
T(P)=\beta P+293 K
$$


Figure 4 b) displays histograms of the measured PO's for three different laser powers. The PO is averaged over eight trapping events all with a maximum mode shift within $\pm 5 \%$ for each power. The normalized shift can be related to a particle position using the top inset of Figure $4 \mathrm{~b}$ ). As expected from equation 4, the higher the power the greater the trap depth and the greater the relative PO towards the bottom of the trap.

Based on these simulations and by adjusting equations 4 and 6 to the measured PO's, the trap strength as defined in equation 5 can be determined, along with an estimate of the temperature elevation of the trapped particle. The solid lines in Figure $4 \mathrm{~b}$ ) correspond to the fit using equation 4 including the Mie theory DoS for $0.25<\Delta \widetilde{\lambda}<1$. The maximum trap strength measured is $6.4 \pm 0.1$. Using equation 5 and the measured polarizability, the intra-cavity intensity is established as $1.3 \mathrm{MW} . \mathrm{cm}^{-2}$ corresponding to an intra-cavity power of $10.4 \mathrm{~mW}$. The maximum values of the longitudinal and transverse spring constants at the bottom of the trap are therefore calculated as $k_{z}=8.9 \times 10^{-3} \mathrm{pN} . \mathrm{nm}^{-1}$ and $k_{x, y}=2 \times 10^{-4}$ pN.nm ${ }^{-1}$. Using the wavefunction maps from Figure 1 a) and the measured trap strength, the mean squared displacement at the maximum power is calculated as $\sigma_{z}=22 \mathrm{~nm}$ and $\sigma_{x, y}=154 \mathrm{~nm}$ (see Supplementary Information for details). The trap strength measured as a function of the intra-cavity power is shown in the inset of Figure $4 \mathrm{~b}$ ). We observe a linear relationship without any marked saturation indicating the absence of over-heating (ie. above $80 \mathrm{~K})$ in the trap, in agreement with the low intra-cavity power measured $(\approx 10 \mathrm{~mW})$. The red line in the inset of Figure 4 b) corresponds to the best fit of equation 6 to the data leading to $\beta \approx 0.4 \mathrm{~K} . \mathrm{mW}^{-1}$ with an upper value of $\beta \approx 4.8 \mathrm{~K} \cdot \mathrm{mW}^{-1}$. Consequently, the temperature elevation can be confidently estimated to be in the range $[0 \mathrm{~K}-50 \mathrm{~K}]$.

\section{Mode-shift time-correlation function}

The spring constants determined above, together with the time autocorrelation function of the mode shift, can be used to determine the friction coefficient, $\gamma$, of the trapped nanoparticles. In the following, we approximate the trapping potential for each laser power as a 


\section{a)}
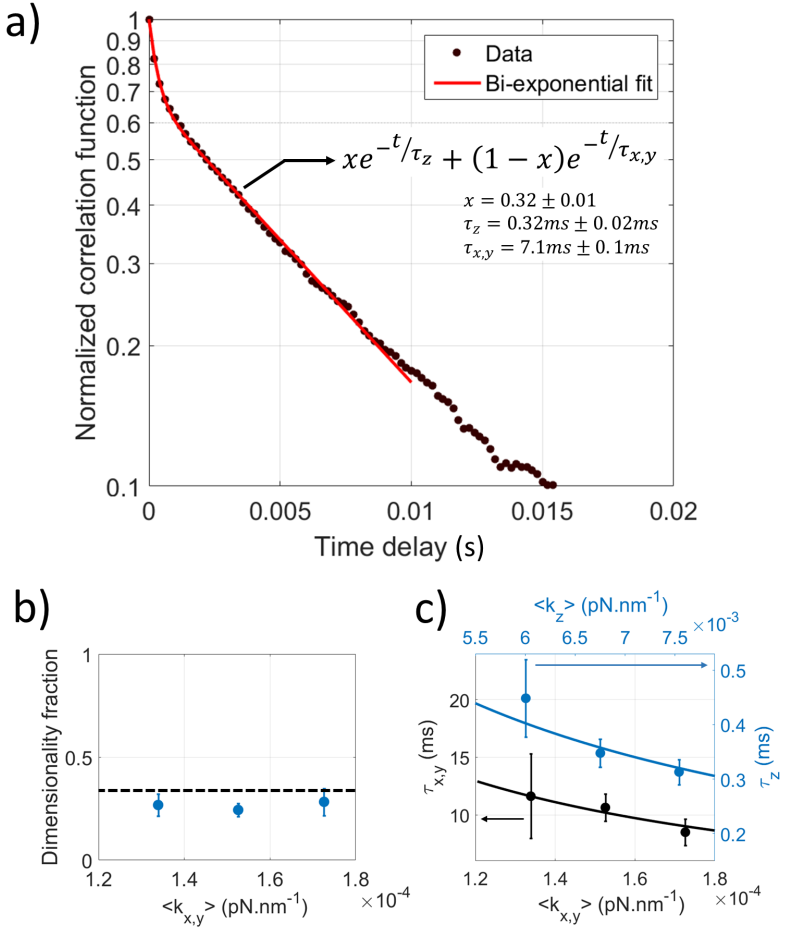

Figure 5: a) Normalized autocorrelation function of the mode shift presented in Figure 1. Black dots are experimental data and red line is a bi-exponential fit. b) Dimensionality factor $x$ as function of the transverse spring constant $\left\langle k_{x, y}\right\rangle$ (blue dots). Theoretical expectation of $1 / 3$ in black dashed line. c) Correlation time constants $\tau_{x, y}$ (black dots) and $\tau_{z}$ (blue dots) as a function of the spring constant $\left\langle k_{x, y}\right\rangle$ and $\left\langle k_{z}\right\rangle$ and their respective fit in solid lines.

harmonic potential characterized by the effective force constants $\left\langle k_{z}\right\rangle$ and $\left\langle k_{x, y}\right\rangle$ (see Supporting Information for details). The force constants can be used to define longitudinal and transverse correlation times, $\tau_{z}=\frac{\gamma_{z}}{2\left\langle k_{z}\right\rangle}$ and $\tau_{x y}=\frac{\gamma_{x y}}{2\left\langle k_{x y}\right\rangle}$. The time autocorrelation function for the mode shift, expressed in terms of these correlation times, is then:

$$
\mathcal{C}_{\Delta \widetilde{\lambda}}(\tau)=x e^{-\tau / \tau_{z}}+(1-x) e^{-\tau / \tau_{x, y}}
$$

with $\tau$ the time delay for autocorrelation and $x=\frac{1}{3}$ the dimensionality factor (see Supporting Information for the derivation of equation 7). The dimensionality factor $x$ describes the relative weight of each spring constant within the autocorrelation function with respect to the number of dimensions the spring constant acts upon. The autocorrelation function for the data presented in Figure $2 \mathrm{~b}$ ) is shown in Figure 5 a)(black circles) together with 
a fit of equation 7 (red line). Applying this fit to the same events used for Figure $4 \mathrm{~b}$ ), we can show that the dimensionality factor is indeed constant with the spring constant in Figure $5 \mathrm{~b}$ ) while the correlation times decrease in Figure $5 \mathrm{c}$ ). The decay constants reveals friction coefficients of $\gamma_{z}=4.8 \pm 0.9 \times 10^{-9} \mathrm{~kg} . \mathrm{s}^{-1}$ along $z$ and $\gamma_{x, y}=3.1 \pm 0.4 \times 10^{-9}$ kg.s ${ }^{-1}$ along $x$ and $y$. These measurements of the friction of coefficient is averaged over the four anti-node positions. For spherical nanoparticles suspended in water, we expect $\gamma=6 \pi \eta r=1.7 \pm 0.4 \times 10^{-9} \mathrm{~kg} . \mathrm{s}^{-1}$, where $\eta$ is the nanoparticle dynamic viscosity in water. In order to compute the friction coefficient in our system, we have used Faxen's law ${ }^{24}$ and the linear superposition assumption ${ }^{25}$ which takes into account the disturbance of the two mirror surfaces to the hydrodynamic properties and then, averaged the result over the four anti-node positions. We finally obtained the values $\gamma_{z}=5.2 \times 10^{-9} \mathrm{~kg} . \mathrm{s}^{-1}$ and $\gamma_{x, y}=2.5$ $\times 10^{-9} \mathrm{~kg} \cdot \mathrm{s}^{-1}$ which are close to the range of measured values including the errorbars.

In conclusion, we have shown that open-access microcavities can be used to trap nanoparticles, providing both in-situ calibration together with sensing capabilities. Measurement of the polarizability along with the trap strength gives direct access to the trap properties without a priori knowledge of the particle. Nanoparticle information such as polarizability, scattering cross-section, friction coefficient, anti-node location, and potentially its absorption cross-section $^{26}$ can also be accessed. Since we have shown that this method can be used even outside of the harmonic approximation, the nanoparticle can be trapped and sensed with a low intra-cavity power, reducing potential damage to biological samples. Such technology opens the way to the realization of a real-time monitoring system for single nanoparticle properties while performing in-situ chemical and biological experiments and avoiding experimental artefacts. 


\section{Methods}

\section{Fabrication and composition of the mirrors}

The open-access microcavity templates are fabricated using a Focused Ion Beam (FIB) in

order to control their topography at the nanometer scale. ${ }^{23}$ The microcavities are situated on a chip which is $300 \mu \mathrm{m} \times 300 \mu \mathrm{m}$ as shown in the Supplementary Information. The mirror coatings comprise Dielectric Bragg Reflector (DBR) stacks centered at a wavelength of 640 $\mathrm{nm}$ and with a reflection bandwidth of $\approx 100 \mathrm{~nm}$, made up of 16 layers of $\mathrm{SiO}_{2} / \mathrm{TiO}_{2}$, with high refractive index for the last layer in order to minimize the mode volume. The mirror coatings were deposited by LaserOptik Gmbh. The highest cavity finesse we have recorded with this coating is $4 \times 10^{4}$, though the finesse is limited by diffraction losses in the work presented here.

\section{Optical setup and data acquisition}

The apparatus allows simultaneous trapping of the particle and measurement of the mode shift $\Delta \lambda$ by scanning the cavity length through resonance with an incident $640 \mathrm{~nm}$ laser. The chip is situated at the centre of a custom-made flow cell, allowing injection of liquids containing polysterene nanoparticles (refractive index $\approx 1.58$ and nominal diameter 200 $\mathrm{nm}$ ) in between the mirrors via inlet and outlet ports (see Supplementary Information and reference $^{27}$ ). For diluted solutions, an event is observed every few minutes, on average.

A $532 \mathrm{~nm}$ laser provides control over the microcavity length by probing the Fabry-Pérot interferences arising at the edge of the DBR stop band. The laser is focused on the flat sections of the chip between the curved mirrors, and its transmission provides real-time length monitoring via a $50 \mathrm{~Hz}$ feedback loop on a DC piezoelectric stack with an accuracy of a few hundreds of picometers. The DC piezoelectric stack controls the cavity length within a $2 \mu \mathrm{m}$ range.

A narrow-linewidth $(<5 \mathrm{MHz} \approx 7 \mathrm{pm})$ continuous wave $639.6 \mathrm{~nm}$ diode laser (Lasos RLK 
DPSS, maximum power of $150 \mathrm{~mW}$ ) is used to address directly a chosen optical mode of the microcavity, and provides the trapping field. Its transmission is recorded on an avalanche photodiode (DET36A/M from Thorlabs) while an AC scanning piezoelectric stack is driven at $5 \mathrm{kHz}$ frequency over a range of 1-2 $\mathrm{nm}$. The APD voltage is visualized and recorded on two oscilloscopes (DPO7104 and TDS2024 both from Tektronix). Once the cavity mode of interest is brought within the scanning range, one of the oscilloscopes monitors the events in real-time, while the second records the data. It has to be noted that the intra-cavity mode

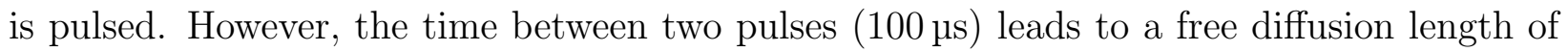
only $20 \mathrm{~nm}$, much smaller than the characteristic lengths of our system. Therefore, we can

reasonably consider the intra-cavity power to be effectively continuous in time. ${ }^{28}$ The laser intensity incident on the reference cavity is lower than that incident on the trapping cavity to preclude trapping of particles in the reference cavity. Any data sets showing evidence of the presence of a nanoparticle in the reference cavity are discarded.

\section{Supporting information}

Supporting information available: Experimental setup and approach, data analysis, position lock technique, Mie theory, FDTD simulations and analytical derivations. This material is available free of charge via the internet at http://pubs.acs.org

\section{Acknowledgements}

A. A. P. Trichet thanks Maxime Richard, Olivier Arcizet and Georgios Alexandrakis for stimulating discussions. This work was supported by the Leverhulme Trust, the Royal Society and the NQIT project.

\section{Competing financial interests}

The authors declare no competing financial interests. 


\section{References}

(1) Foreman, M. R.; Swaim, J. D.; Vollmer, F. Adv. Opt. Photonics 2015, 7, 168-240.

(2) Baaske, M.; Vollmer, F. ChemPhysChem 2012, 13, 427-436.

(3) Vollmer, F.; Arnold, S. Nat. Methods 2008, 5, 591-596.

(4) Vollmer, F.; Arnold, S.; Keng, D. Proc. Natl. Acad. Sci. U. S. A. 2008, 105, 2070120704 .

(5) Zhu, J.; Ozdemir, S. K.; Xiao, Y.-F.; Li, L.; He, L.; Chen, D.-R.; Yang, L. Nat. Photonics 2010, 4, 46-49.

(6) Zhu, J.; Özdemir, Ş. K.; He, L.; Chen, D.-R.; Yang, L. Opt. Express 2011, 19, 1619516206.

(7) Zijlstra, P.; Paulo, P. M.; Orrit, M. Nat. Nanotechnol. 2012, 7, 379-382.

(8) Juan, M. L.; Righini, M.; Quidant, R. Nat. Photonics 2011, 5, 349-356.

(9) Daly, M.; Sergides, M.; Nic Chormaic, S. Laser Photonics Rev. 2015, 9, 309-329.

(10) Grigorenko, A.; Roberts, N.; Dickinson, M.; Zhang, Y. Nat. Photonics 2008, 2, 365370.

(11) Descharmes, N.; Dharanipathy, U. P.; Diao, Z.; Tonin, M.; Houdré, R. Lab Chip 2013, $13,3268-3274$.

(12) Kotnala, A.; DePaoli, D.; Gordon, R. Lab Chip 2013, 13, 4142-4146.

(13) Zehtabi-Oskuie, A.; Bergeron, J. G.; Gordon, R. Sci. Rep. 2012, 2.

(14) Zhang, W.; Huang, L.; Santschi, C.; Martin, O. J. Nano Lett. 2010, 10, 1006-1011.

(15) Al Balushi, A. A.; Kotnala, A.; Wheaton, S.; Gelfand, R. M.; Rajashekara, Y.; Gordon, R. Analyst 2015, 
(16) Al Balushi, A. A.; Gordon, R. ACS Photonics 2014, 1, 389-393.

(17) Kang, P.; Schein, P.; Serey, X.; ODell, D.; Erickson, D. Sci. Rep. 2015, 5.

(18) Juan, M. L.; Gordon, R.; Pang, Y.; Eftekhari, F.; Quidant, R. Nat. Phys. 2009, 5, $915-919$.

(19) Descharmes, N.; Dharanipathy, U. P.; Diao, Z.; Tonin, M.; Houdré, R. Phys. Rev. Lett. 2013, 110, 123601.

(20) Neumeier, L.; Quidant, R.; Chang, D. E. New J. Phys. 2015, 17, 123008.

(21) Wheaton, S.; Gordon, R. Analyst 2015, 140, 4799-4803.

(22) Dolan, P. R.; Hughes, G. M.; Grazioso, F.; Patton, B. R.; Smith, J. M. Opt. Lett. 2010, $35,3556-3558$.

(23) Trichet, A. A. P.; Dolan, P. R.; Coles, D. M.; Hughes, G. M.; Smith, J. M. Opt. Express 2015, 23, 17205-17216.

(24) Schäffer, E.; Nørrelykke, S. F.; Howard, J. Langmuir 2007, 23, 3654-3665.

(25) Lin, B.; Yu, J.; Rice, S. A. Phys. Rev. E 2000, 62, 3909.

(26) Mader, M.; Reichel, J.; Hänsch, T. W.; Hunger, D. Nat. Commun. 2015, 6.

(27) Trichet, A. A. P.; Foster, J.; Omori, N.; James, D.; Dolan, P.; Hughes, G.; Vallance, C.; Smith, J. Lab Chip 2014, 14, 4244-4249.

(28) Deng, Y.; Bechhoefer, J.; Forde, N. R. J. Opt. A: Pure Appl. Opt. 2007, 9, S256. 